\title{
Cross-cultural Adaptation of the Body Satisfaction and Global Self Perception Questionnaire for Subjects with Non-specific Musculoskeletal Disorders
}

\author{
Gemma V. Espí-López 1,\#,*, Lucas Monzani2 ${ }^{2, \#, ~ R o s a r i o ~ Z u r r i a g a ~ 3, \#, ~}$ \\ Pierre-Michel Dugailly ${ }^{4}$, M. Teófila Vicente-Herrero ${ }^{5}$, Lars L. Andersen ${ }^{6}$ \\ 'Department of Physiotherapy, Faculty of Physiotherapy, University of Valencia, Gascó \\ Oliag Street, 5, 46010 Valencia, Spain \\ 2Ivey Business School, Western University, Canada \\ 3 University Research Institute of Human Resources Psychology, Organizational \\ Development and Quality of Work Life (IDOCAL), University of Valencia, Spain \\ ${ }^{4}$ Research Unit in Osteopathy, Faculty of Motor Sciences, Université Libre de Bruxelles \\ (ULB), Brussels, Belgium \\ ${ }^{5}$ Occupational Health Service, Correos Group of Valencia, Spain \\ ${ }^{6}$ National Research Centre for the Working Environment, DK-2100 Copenhagen, \\ Denmark \\ *The authors have contributed equally
}

\section{Article Type: Article \\ Article Citation: Gemma V. \\ Espí-López, Lucas Monzani, Rosario \\ Zurriaga, Pierre-Michel Dugailly, \\ Teófila Vicente-Herrero M, Lars L. \\ Andersen. Cross-cultural adaptation \\ of the body satisfaction and global self perception questionnaire for subjects with non-specific musculoskeletal disorders. Indian Journal of Science and Technology. 2020; 13(07), 817-831. D01: 10.17485/ijst/2020/v013i07/149850}

Received date: January 8, 2020

Accepted date: January 29, 2020

*Author for correspondence: Gemma V. Espí-López gemma. espi@uv.es $\bigcirc$ Department of Physiotherapy, Faculty of Physiotherapy, University of Valencia, Gascó Oliag Street, 5, 46010 Valencia, Spain

\begin{abstract}
Background: Assessing patient's cognitions and emotions about their physical body is an important part of rehabilitation planning, as musculoskeletal disorders can lead to a change in bodily perceptions. Methods: This study explores the validity and reliability of the Body Satisfaction and Global Self-Perception Questionnaire (QSCPGS)] in the Spanish population. In addition to calculating Cronbach's alpha, we conducted Exploratory Factor Analysis and Confirmatory Factor Analyses to test the scale's validity. We then conducted a test-retest and longitudinal measurement invariance test to explore our measure's reliability. Results: Our final sample consisted of 147 subjects with non-specific musculoskeletal disorders $(M=32$ years, $S D=15.06)$, who provided two data points in a 30-day period. A reduced, four-factor model fit the data better $\left[\left(X^{2}=92.51^{*} ; \mathrm{df}=\right.\right.$ $71 ; \mathrm{df}=\mathrm{X}^{2} / \mathrm{df}=1.30 ; \mathrm{RMSEA}=.04 ; \mathrm{CFI}=.95 ; \mathrm{TLI}=.94 ; \mathrm{SRMR}=.05 ;$ WRMR $=.84)]$ than any other model. The re-test validity analyses revealed that the four-factor model was stable over time. Finally, the reduced scale correlated with the SF-36 Quality of Life inventory and participants' BMI. Conclusions: The QSCPGSe (Spanish version QSCPGS) is a reliable and effective tool for measuring body image perceptions that are more accurate than the original scale.
\end{abstract}

Keywords: Body Satisfaction, Questionnaire, Global Self Perception, Musculoskeletal. 


\section{Background}

Musculoskeletal disorders, such as chronic back pain [1] or musculoskeletal degenerative disorders [2-3] as well as pain and postural alterations can affect how people perceive and feel about their body image (body perceptions and sensations, respectively) [4-8]. Given that such alterations are both cognitive and affective in nature, incorporating insights from clinical psychology research on body sensations and perceptions can be a useful practice when designing interventions aimed at treating musculoskeletal problems. However, whereas several scales to measure body satisfaction exist, such scales were primarily developed for people with eating disorders and thus, focus on the physical image (perception) but not on body sensation [9].

The experience of bodily (dis)comfort its multi-factorial nature, and thus difficult to quantify purely relying on physiological indicators. Thus, to be efficient, a measure of body image requires the insights from physio and psychotherapists to be useful in the differential diagnosis and treatment of body image disorders. To create such a measure, Evers and Verbancka [10] developed the Questionnaire de Satisfaction Corporelle et de Perception Globale de Soi (QSCPGS), a self-report questionnaire that captures "body satisfaction and global self-perception". The French version of the QSCPGS consists of 20 items divided into two blocks of 10 items each. The first item block measures how the individual perceives his or her body ("Body"; body perceptions) and the second item block measures individuals' feelings towards their body ("Feelings"; body sensations). Some initial evidence of validity for QSCPGS for the French population exists, but the scale neither has been translated to other languages, nor validated in other populations. Thus, given its value as an inter-disciplinary instrument, the translation of the QSCPGS into other languages and its validation populations are both urgent and important endeavours.

The QSCPGS has several issues that require a revision of its psychometric properties. First, an in-depth look of the original validation article suggests the development of the QSCPGS was completely a-theoretical. In other words, the authors claim adapting items from established scales in psychology such as the STAI and Rosenberg's depression scale without any sound theorizing of how these items might relate to each other, or its underlying mechanisms. In other words, a solid rationale explaining why "Body" and "Feelings" are its main dimensions is missing. Thus, a first goal of this work is exploring the structure and relations of the QSCPGS dimensions.

Second, in their study, Evens and Verbancka provided very limited evidence about the psychometric properties of theQSCPGS. For example, Evens and Verbancka only reported the Cronbach's alpha of the overall scale. However, without any evidence that both dimensions of the QSCPGS's load into a higher-order construct (e.g., "Body Image"), the aggregation of all items into a single construct is methodologically incorrect and thus calculating Cronbach's alpha for an overall construct might be misleading (i.e., inflating the reliability score). Consequently, the second goal of this work is to conduct an in-depth examination of the psychometric properties of the QSCPGS.

Finally, Evens and Verbancka provided very limited evidence of what is the predictive validity and reliability of the QSCPGS. Again, in their study, these authors only provide some evidence that a higher score in the QSCPGS increases relaxation in patients with 
clinical depression. Thus, testing how the QSCPGS relates to established measures of overall health (e.g., the SF-36), or accepted physiological indicators related to "Body Image" (e.g., BMI) is an absolute necessity to ensure that the QSCPGS is a valuable addition to collection of existing measures of "Body Image". Thus, the third goal of this study is to explore the predictive validity and reliability of the QSCPGS on well-established metrics, in a sample of patients suffering of musculoskeletal disorders.

\section{Materials \& Methods}

\subsection{Sample}

Our sample included people with chronic, non-specific, musculoskeletal disorders (cervical, dorsal, lumbar, upper limbs, lower limbs), which were diagnosed and treated in two primary care centers in Valencia (Spain). The exclusion criteria were (a) reporting pain due to other non-musculoskeletal disorders; (b) physical or mental disability; and (c) or be receiving different medical treatment than the usual.

The same questionnaires were applied twice with four weeks in between. The sample in the first round consisted of 176 people. In the second round, 150 of these completed our questionnaires. After removing cases with missing data at times 1 and 2, the final sample consisted of 147 participants (see Table 1).

\subsection{Procedure}

This study consisted of three steps. First, a transcultural translation was carried out. Second, the translated questionnaire was applied to a sample of the Spanish population. Third, a factorial analysis of the questionnaire was conducted, and for exploratory purposes only, the questionnaire was correlated with variables of the 36-item Short Form Health Survey Questionnaire (SF-36) (General Health, Role-physical, Role-emotional and Physical Function).

Prior to completing the questionnaires, a structured interview was conducted that included questions regarding the practice of physical activity and anthropometric data to calculate the Body Mass Index (BMI), fulfilling all confidentiality procedures in accordance with the Declaration of Helsinki. In May 2016, the creators of the questionnaire authorized to carry out the translation and to provide evidence about the validity of the QSCPG in a Spanish sample (see Figure 1). Informed written consent was obtained from all the participants prior to inclusion and ethics approval has been received from the University of Valencia (H1512328491077).

The sample size was obtained following the guidelines by [11], assuming a level of bilateral significance of $\alpha=0.05$ and a statistical power of $80 \%$. To compensate for possible losses or dropouts, a minimum size of 126 subjects was established.

\subsection{Measures}

Questionnaire de Satisfaction Corporelle et de Perception Globale de Soi (QSCPGS): In their original work, Evers \& Verbanch's scale consists of two factors ("Body" and "Feelings"). 
Cross-cultural Adaptation of the Body Satisfaction and Global Self Perception Questionnaire for Subjects with Non-specific Musculoskeletal Disorders

TABLE 1. Sample characteristics

\begin{tabular}{|c|c|c|c|c|c|c|}
\hline & \multicolumn{3}{|c|}{$n=176$} & \multicolumn{3}{|c|}{$n=147$} \\
\hline & Range & Mean & SD & Range & Mean & SD \\
\hline Age & $18-76$ & 32.52 & 15.06 & $18-76$ & 32.49 & 14.74 \\
\hline Weight (kg) & $46-118$ & 66.04 & 12.56 & $47-118$ & 66.46 & 12.61 \\
\hline \multirow[t]{2}{*}{ Height $(\mathrm{cm})$} & $150-200$ & 169.06 & 9.56 & $150-200$ & 169.46 & 9.79 \\
\hline & $n$ & \multicolumn{2}{|c|}{$\%$} & $n$ & \multicolumn{2}{|r|}{$\%$} \\
\hline \multicolumn{7}{|c|}{ Gender } \\
\hline Female & 105 & \multicolumn{2}{|c|}{59.3} & 84 & \multicolumn{2}{|r|}{57.1} \\
\hline Male & 71 & \multicolumn{2}{|c|}{40.1} & 63 & \multicolumn{2}{|r|}{42.9} \\
\hline \multicolumn{7}{|c|}{ Studies } \\
\hline Junior High School & 21 & \multicolumn{2}{|c|}{11.9} & 21 & \multicolumn{2}{|r|}{14.3} \\
\hline Professional Training & 6 & \multicolumn{2}{|c|}{3.4} & 5 & \multicolumn{2}{|r|}{3.4} \\
\hline High School & 7 & \multicolumn{2}{|c|}{4.0} & 6 & \multicolumn{2}{|r|}{4.1} \\
\hline University & 118 & \multicolumn{2}{|c|}{66.7} & 105 & \multicolumn{2}{|r|}{71.4} \\
\hline \multicolumn{7}{|c|}{ Occupation } \\
\hline Public official & 57 & \multicolumn{2}{|c|}{32.2} & 51 & \multicolumn{2}{|r|}{34.7} \\
\hline Employee & 12 & \multicolumn{2}{|c|}{6.8} & 11 & \multicolumn{2}{|r|}{7.5} \\
\hline Freelancer & 6 & \multirow{2}{*}{\multicolumn{2}{|c|}{3.4}} & 3 & \multicolumn{2}{|r|}{2} \\
\hline Unemployed & 29 & & & 14 & \multicolumn{2}{|r|}{9.5} \\
\hline Student & 72 & \multicolumn{2}{|c|}{40.7} & 68 & \multicolumn{2}{|r|}{46.3} \\
\hline \multicolumn{7}{|c|}{ Physical activity } \\
\hline Yes & 94 & & & 80 & & 54.4 \\
\hline No & 82 & & & 67 & & 45.6 \\
\hline & & Pai & cation & & & \\
\hline Changing & 12 & & & 9 & & 6.1 \\
\hline Cervical & 67 & & & 55 & & 37.4 \\
\hline Dorsal & 21 & & & 17 & & 11.6 \\
\hline Lumbar & 40 & & & 35 & & 23.8 \\
\hline Upper limbs & 14 & & & 13 & & 8.8 \\
\hline Lower limbs & 22 & & & 18 & & 12.2 \\
\hline
\end{tabular}

These authors report that Cronbach's alpha for the overall scale was of $\alpha=.83$ in sample of 54 participants. No further psychometric information was provided.

We used Brislin's guidelines to translate the original questionnaire into Spanish [12] (see Annex I for how consensus was reached; Annex II and III respectively for the Spanish and English version of the QSCPGS items).

SF-36: We used the Spanish adaptation of the SF-36 questionnaire, which measures physical and mental health [13]. The Spanish adaptation of the SF-36 showed high validity and reproducibility in prior studies [14]. The SF-36 consists of 36 items comprising eight dimensions (Bodily Pain, Perceptions, Mental Health, Physical Function, Role-physical, Role-emotional, Social Function, and Vitality). To assess each dimension, items are aggregated into a score ranging from 0 to 100; being higher score indicators of good health [15]. 


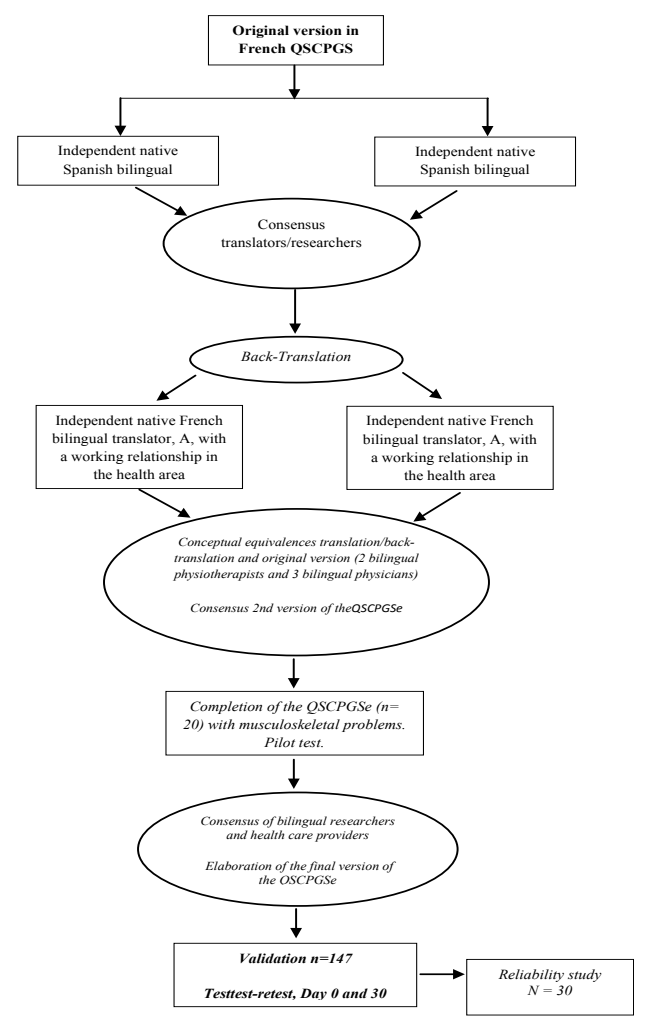

FIGURE 1. Steps in the French-Spanish adaptation process.

For this study, we considered the following dimensions of the questionnaire that are more related to the QSCPGS items: General Health, Role-Physical, Role-Emotional and Physical Function. General Health is evaluated with the item "In General, would you say your health is:" with alternative answers ranging from $1=$ Excellent to $5=$ Poor. Role-physical consists of 4 items related to physical health-caused problems at work or in carrying out daily activities. Item example: "Have you had any of the following problems with your work or other regular daily activities?" The response alternatives go from $1=$ always to $5=$ never; Role-emotional composed of 3 items related to problems at work in daily activities due to an emotional problem. Item example "Have you had any of the following problems with your work or other regular daily activities as a result of any emotional problems?”. The reply alternatives go from 1 = always to 5 = never; Physical Function comprises items 7: "How much bodily pain have you had during the past 4 weeks?" and 8 "Over the last 4 weeks how much did pain interfere with your normal work?". The reply alternatives go from $1=$ none/ not at all to $7=$ a lot.

\subsection{Data Analysis}

We conduct our analyses using SPSS24.0 and MPLUS 7.2 [16]. We calculated descriptive and internal reliability statistics, followed by a set of exploratory (EFA) and confirmatory factor analyses (CFA). First, we used EFA to examine the distribution patterns and 
the underlying components of the 20 items, employing a Principal Axis Method and a Varimax rotation. We calculated the value of the Kaiser-Meyer-Olkin sample adequacy measurement (KMO) and Bartlett's test of Sphericity [17].

For CFAs, we used a robust estimation method, the Maximum Likelihood Mean and Variance adjusted (MLSMV), which ensures that the results are not affected by deviations from multivariate normality [18]. We assessed our models' fit using the Chi-Square/ degrees of freedom ( $\chi 2 / \mathrm{df})$, comparative fit index (CFI), Tucker's fit index(TFI), root mean square error of approximation (RMSEA), standardized root mean residual (SRMR), and Weighted root mean residual (WRMR) [19]. Finally, we tested the reliability factors using Cronbach's $\alpha$ and Raykov's $\tau$.

To evaluate test-retest reliability of the reduced scale, we deployed a three-fold analytical strategy. First, we conducted a follow-up measurement of the QSCPGS questionnaire on all participants one month after the initial measurement. We excluded from our analysis any participant with missing data at Time 2. Following the recommendations of [20], we calculated the relative reliability of the scale using an Intra-class Correlation (ICC) coefficient, specifying mixed-effects, two-way model and a consistency type for each factor of the QSCPGS.

Second, we calculated the absolute reliability by calculating the Standard Error of Measurement (SEM) and the Minimal Detectable Change (MCD) [21], alongside paired samples $t$-test for each of the factors of the QSCPGS. Non-significant scores would suggest that there was no change in patient's scores. Furthermore, given that we expect these latent factors to be correlated, we tested the longitudinal scalar invariance of the QSCPGS factors. Model A tested a four-factor model vs the original two-factor model, and a singlefactor model (Time 1). Model B replicated our CFA in a second dataset (Time 2).

Finally, we conducted a longitudinal scalar invariance test (LSI) to test the temporal stability of the latent factorial structure (dimension) of our revised scale. The LSI goes beyond a typical metric invariance test, by requiring that the intercepts to be also equivalent across administrations. Thus, in Model C, we set item loadings to equivalence and constrained the factors identified in Model A to "0".A good fitting model would suggest that there is no temporal change in the latent factors' estimated means.

\section{Results}

In the first dataset, the KMO value was .79 and Barlett's test of sphericity was significant $(p<.0001)$ showing very good sampling adequacy. After rotating the factor matrix, six factors explained $64.68 \%$ of the variance. However, we excluded items loading in factor 6 , due to poor fit with the two original scale's factors. The remaining five factors explained $59.05 \%$ of the variance (see Table 2 ).

Based on the results of our EFA, we retained four factors. A first factor, labelled "Vitality", which contained three items with factor loadings ranging between .77 and .46. The second factor, labelled "Aesthetic Appraisal", contained three items with factor loadings ranging between .77 and .46. The third factor, "Cognitive Discomfort", contained two items with factor loadings ranging between .75 and .67. The fourth factor comprised 
TABLE 2. Exploratory factor analysis -test $(\mathrm{N}=177)$

\begin{tabular}{|c|c|c|c|c|c|c|}
\hline & 1 & 2 & 3 & 4 & 5 & 6 \\
\hline Item 11: Feel-1: Weak-Strong & .77 & & & & & \\
\hline Item 13: Feel-3: Empty-Full & .64 & & & & & \\
\hline Item 19: Feel-9: Careless-Careful & .57 & & & & & \\
\hline Item 16: Feel-6: Tired-Rested & .56 & & & & & \\
\hline Item 5: Body-5: Timid-Audacious & .53 & & & & & \\
\hline Item 15: Feel-5: Inferior-Superior & .46 & & & & & \\
\hline Item 2: Body-2: Attractive-Repulsive & & .98 & & & & \\
\hline Item 3: Body-3: Source of: Pleasure-Displeasure & & .84 & & & & \\
\hline Item 4: Body-4: Pure-Impure & & .53 & & & & \\
\hline Item 1: Body-1: Unhealthy-Healthy & & .47 & & & & \\
\hline Item 8: Body-8: Angry-Peaceful & & & .75 & & & \\
\hline Item 7: Body-7: Cold-Warm & & & .67 & & & \\
\hline Item 10: Body-10: Old-Young & & & & - & & \\
\hline Item 14: Feel-4: Free to Act - Constrained & & & & .65 & & \\
\hline Item 12: Feel-2: Happy-Sad & & & & .49 & & \\
\hline Item 9: Body-9: Calm-Nervous & & & & & -.69 & \\
\hline Item 18: Feel-8: Impatient-Patient & & & & & .52 & \\
\hline Item 20: Feel-10: Good-Bad & & & & & & .62 \\
\hline Item 17: Feel-7: Worthy-Worthless & & & & & & .56 \\
\hline Item 6: Body-6: Feminine-Masculine & & & & & & - \\
\hline
\end{tabular}

Note: The extraction method was a principal axis factoring (Waldman, 1985). A varimax rotation with Kaiser Normalization was applied to extracted factor matrix. Items were sorted based on their loadings and for parsimony scores below .40 are not shown.

items with factor loadings ranging between .65 and .49 , whereas the fifth factor included two semantic differentials, with factor loadings ranging between .61 and .56. Given that few items loaded on factors 4 and 5, we merged factors 4 and 5 into a single factor, with two items, labeled "Affective Discomfort". We used these factor loadings to test a reduced version of the scale comprising 10 items (see Table 3).

Table 3 shows SEM\% < 10\% was obtained for Vitality (9\%), and it was low for Affective (Dis)comfort (12\%) and Aesthetic Appraisal (17\%) and moderate for Cognitive Discomfort (27\%). MDC $_{95} \%$ was $\leq 30 \%$ for Vitality (24\%). Affective (Dis) comfort was barely above the $\leq 30 \%$ cutoff value (34\%). Not surprisingly, Aesthetic appraisal (46\%) and Affective discomfort (74) were substantially above the $\leq 30 \%$ threshold for $\mathrm{MDC}_{95} \%$.

Similarly, CFAs revealed that the four-factor models A and B presented a better fit than a single- or two-factor models (no convergence). Moreover, all dimensions of the fourfactor model presented adequate reliabilities (Cronbach' $\alpha=68$ and Raykov's $\tau=.76$ for the "Vitality" factor'; Cronbach's $\alpha=.84$ and Raykov's $\tau=.87$ for the "Aesthetic Appraisal" factor; Cronbach's $\alpha=.74$ and Raykov's $\tau=.74$ for the "Cognitive Discomfort" factor; Cronbach's $\alpha=.70$ and Raykov's $\tau=.72$ for the "Affective Discomfort" factor). Finally, Model C revealed that items 12 and 14 presented differential item functioning (DIF) and thus were excluded from our scale. In addition, we removed items 15 and 16 as result of a poor loading in CFA's model A and B (Table 2).

In the four-factor model, all factor loadings were statistically significant and above the .50 cutoff value. The LSI model also showed an appropriate fit, with the exception of 
Cross-cultural Adaptation of the Body Satisfaction and Global Self Perception Questionnaire for Subjects with Non-specific Musculoskeletal Disorders

TABLE 3. Confirmatory factor analysis, model fit, and Goodness-of-fit indicators (N $=147)$

\begin{tabular}{|c|c|c|c|c|c|c|c|c|}
\hline $\begin{array}{l}\text { Model A - Test } \\
\text { (T1) }\end{array}$ & $X^{2}$ & Df & $\mathrm{X}^{2} / \mathrm{df}$ ratio & RSMEA & CFI & TLI & SRMR & WRMR \\
\hline Null Model & 353.82 & 45 & 7.86 & - & - & - & - & - \\
\hline 4-Factor & 32.71 & 29 & 1.12 & .03 & .99 & .98 & .04 & .07 \\
\hline $\begin{array}{l}\text { Model B - } \\
\text { Re-test (T2) }\end{array}$ & $X^{2}$ & Df & $\mathrm{X}^{2} / \mathrm{df}$ ratio & RSMEA & CFI & TLI & SRMR & WRMR \\
\hline Null Model & 414.50 & 91 & 4.55 & - & - & - & - & - \\
\hline 4-Factor & $54.07^{\star}$ & 29 & 1.86 & .08 & .91 & .86 & .07 & 1.12 \\
\hline Model C - LSI & $X^{2}$ & Df & $\mathrm{X}^{2} / \mathrm{df}$ ratio & RSMEA & CFI & TLI & SRMR & WRMR \\
\hline Null Model & $728.55^{\star * *}$ & 190 & 3.83 & - & - & - & - & - \\
\hline 4-Factor & $184.38^{\star}$ & 154 & 1.20 & .04 & .94 & .93 & .06 & .96 \\
\hline
\end{tabular}

Note: ${ }^{* *} p<.001 ;{ }^{* *} p<.01$. The estimator used was a Minimum Least Squares Mean and Variance adjusted (MLMV) with a convergence limit set at 1000 interactions. Neither a single factor, nor a two-factor model converged in our analyses. LSI = Longitudinal Scalar Invariance.

the Tucker Lewis index (TLI) which was barely 90 (Table 3). The change in the factors' estimated means was non-significant except for "Vitality" $(\Delta \mathrm{EM}=.31 ; p<.05)$. These results initially support the QSCPGSe's LSI (Figures 2 and 3).

Pearson was computed to analyze associations between the QSCPGSe, BMI, and the potentially related dimensions of the SF-36. The factor I "Vitality" correlated positively and significantly with "Role-Physical" $\left(\mathrm{r}=.184^{*}\right)$ and "Role-Emotional" $\left(r=.252^{\star *}\right)$. The factor II "Aesthetictic appraisal" was associated positively and significantly with BMI ( $r=$

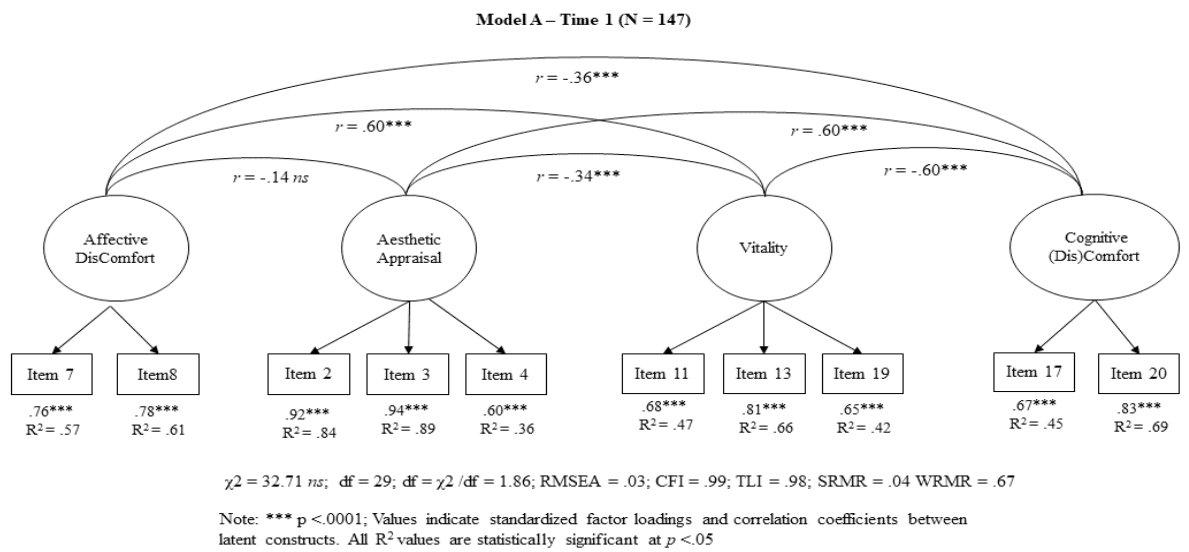

Model A: $\chi 2=32.71 \mathrm{~ns} ; \mathrm{df}=29 ; \chi 2 / \mathrm{df}=1.12 ; \mathrm{RMSEA}=.03 ; \mathrm{CFI}=.99 ; \mathrm{TLI}=.98 ; \mathrm{SRMR}=.04 ; \mathrm{WRMR}=.07$

Note: ${ }^{* * *} \mathrm{p}<.0001$; Values indicate standarized factor loadings and correlation coefficients between laten constructs. All $\mathrm{R}^{2}$ values are statistically significant at $\mathrm{p}<.05$

FIGURE 2. Test - confirmatory factor analysis for the revised scale (four-factor model) 


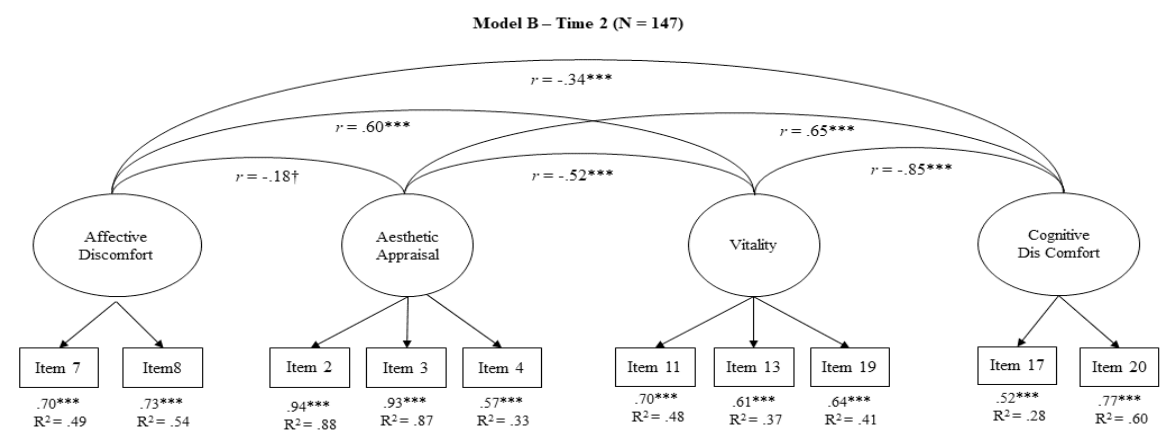

Model B: $\chi^{2}=54.07^{*} ; \mathrm{df}=29 ; \chi^{2} / \mathrm{df}=1.86 ; \mathrm{RMSEA}=.08 ; \mathrm{CFI}=.91 ; \mathrm{TLI}=.86 ; \mathrm{SRMR}=.07 ; \mathrm{WRMR}=1.11$

Note: ${ }^{* *} p<.0001$; Values indicate standarized factor loadings and correlation coefficients between laten constructs. All $R^{2}$ values are statistically significant at $p<.05$

FIGURE 3. Re-test - confirmatory factor analysis for the revised scale (four-factor model).

$\left..342^{\star *}\right)$ and "General Health" $\left(r=.366^{* \star}\right)$. The "Cognitive Discomfort" factor (Factor III) failed to correlate significantly with any SF-36 factor. Finally, the fourth factor "Affective Discomfort" correlated negatively and significantly with the factor "Role-Emotional" of questionnaire SF-36 $\left(r=-.325^{\star *}\right)$.

\section{Discussion}

The goals of the present study were (a) to provide a conceptual framework for the QSCPGS; (b) to test the psychometric properties of the QSCPGS in the Spanish population; and (c) to test its predictive validity by exploring its relationship with outcomes of interest (e.g., BMI, Overall health).

Regarding the first objective, our findings revealed that a revision of the original scale was necessary. Figure 4 shows our proposed framework as suggested by our findings. The revised framework now enables physicians to screen better their patients and tailor their treatment. More importantly, the revised version facilitates inter-disciplinary work between physio and psychotherapists in what regards to body image.

Regarding our second objective, this study provides practitioners with a revised version of the QSCPGS, the QSCPGSe (see Annex I for a Spanish version and Annex II for an English version). The QSCPGSe has good psychometric properties, and a higher degree of accuracy regarding the underlying facets of a person's body image perceptions and sensations. Thus, our study shows initial evidence about the construct validity of the QSCPGSe. Furthermore, the QSCPGSe has good internal reliability and validity, acceptable test-retest validity and longitudinal scalar invariance. Moreover, the QSCPGSe showed the convergent validity as it correlated with other scales measuring similar constructs (SF36). Finally, we significantly reduced the number of items that compose the QSCPGSe scale, which facilitates its application alongside other questionnaires.

This work provides an important contribution to the body image field, as it helps to structure and thus advance our understanding individuals' perceptions and sensations 


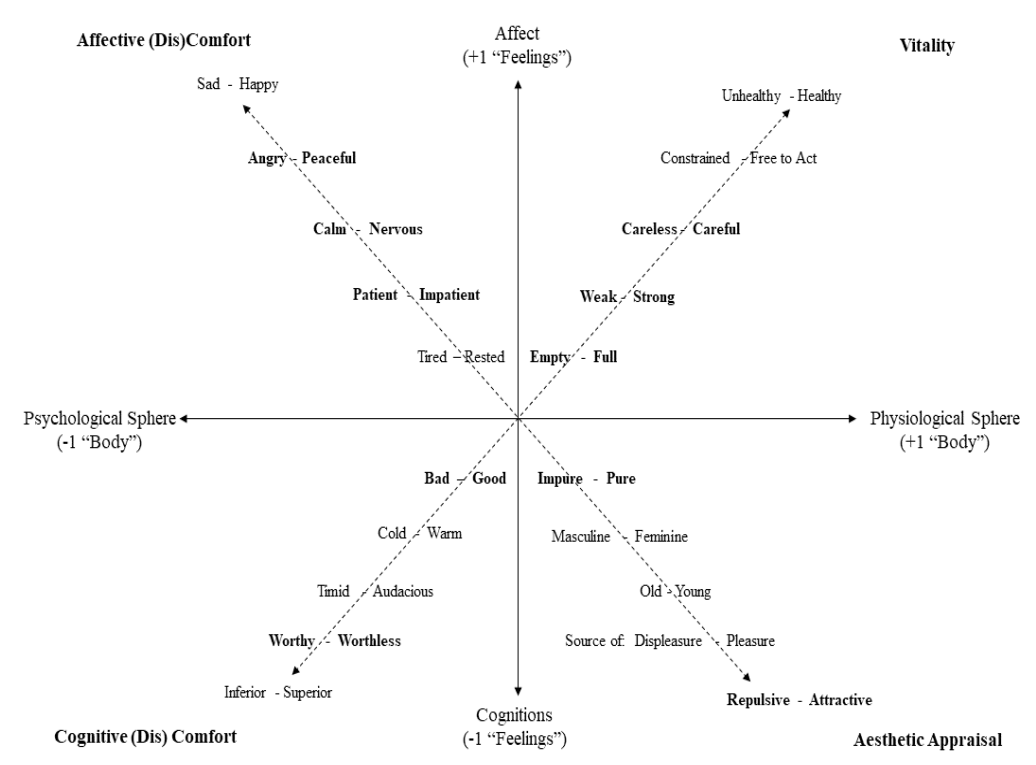

FIGURE 4. Theoretical model for inter-disciplinary assessment of body image perceptions.

of their body image when structural or functional suffering is present. For example, the QSCPGSe enhances our understanding of body image by unpacking bodily self-perception into four more specific factors. Of these 4 factors, 2 correspond to high and low scores of the "body" factor and high and low scores of the "feelings" factor.

The new version includes the factors vitality, aesthetic appraisal, affective discomfort and cognitive discomfort, whereby it is possible to detect areas in which the subject may offer diminished values and not solely collect an overall sum of self-perception, as occurs with other questionnaires such as the SF-36. Furthermore, there are other questionnaires specific to pathologies or quality of life and health that include physical and emotional subscales with which the questionnaire of this study could expand results and offer relevant information about subjects such as the Nottingham Health Profile [22], Euro-QOL [23], PTOPS-I [24], Michigam Hand Outcomes Questionnaire (MHQ-Thai) [25], or the Nordic Musculoskeletal Questionnaire (NMQ) [26].

The present study also has implications for practice. First, there are virtually no questionnaires for guidance in the holistic assessment of overall body perception in subjects with musculoskeletal or other disorders. This questionnaire can be used not only for musculoskeletal pathologies, but also for any disease, and even more so in chronic cases. Moreover, given its short form, it can be used to screen patients alongside questionnaires assessing anxiety, depression, musculoskeletal, visceral, neurological or orthopaedic pathologies, health and quality of life, providing valuable information on the individual's condition at many levels and in numerous areas. Furthermore, it offers the possibility of discovering essential elements in different populations regarding the age or gender associated with certain corporal dissatisfaction, as suggested by the authors of the original questionnaire [10]. Moreover, our study is particularly relevant for health professionals 
concerned with chronic pain, given the lack of specific measures available to capture body image perceptions in subjects with musculoskeletal disorders, or other forms of chronic pain. Future studies should consider validating this revised scale in other countries.

Our study has certain limitations. First, our instrument was only validated in an Iberian sample, and not in other Spanish speaking populations. Future studies should test it in other Spanish speaking regions (e.g., Latin America) or in English speaking populations (e.g., Canada). Similarly, as result of scale reduction process, two factors have less than 3 items, which is the usual recommendation when constructing psychometric scales [17]. Future studies should attempt to extend our work using three indicators per factor. However, despite these limitations, our work provides practitioners with a reliable and effective instrument to measure body image perception.

\section{Conclusions}

There are virtually no questionnaires that provide guidance for the interdisciplinary assessment and differential diagnosis of overall body perception in patients with musculoskeletal pain. The QSCPGSe can be used to diagnose the impact of musculoskeletal pathologies on mental health (and vice versa); even more so in chronic cases of musculoskeletal pain or psychological symptoms (e.g., Anxiety and Depression). The QSCPGSe shows good psychometric properties in a Spanish population, and a much higher degree of accuracy than the original scale. The QSCPGSe has a significantly lower number of items than the original scale. This reduced number of items simplifies its use in a stand-alone mode, or its combination with other surveys. In conclusion, the QSCPGSe can be administered alongside questionnaires assessing anxiety, depression, musculoskeletal, visceral, neurological or orthopedic pathologies, and overall health and quality of life (e.g., SF-36).

\section{Acknowledgements}

The authors appreciate with gratitude all the patients who selflessly participated in the study.

\section{References}

1. Moseley GL. I can't find it! Distorted body image and tactile dysfunction in patients with chronic back pain. Pain. 2008, 140(1), 239-243. DOI: 10.1016/j.pain.2008.08.001.

2. Jorge RTB, Brumini C, Jones A, Natour J. Body image in patients with rheumatoid arthritis. Modern Rheumatology. 2010, 20(5), 491-495. DOI: 10.1007/s10165-010-0316-4.

3. Lotze M, Moseley GL. Role of distorted body image in pain. Current Rheumatology Reports. 2007, 9(6), 488-496. DOI: 10.1007/s11926-007-0079-x.

4. Brumagne S, Janssens L, Janssens E, Goddyn L. Altered postural control in anticipation of postural instability in persons with recurrent low back pain. Gait \& Posture. 2008, 28(4), 657662. DOI: 10.1016/j.gaitpost.2008.04.015. 
5. Hodges PW, Richardson CA. Altered trunk muscle recruitment in people with low back pain with upper limb movement at different speeds. Archives of Physical Medicine and Rehabilitation. 1999, 80(9), 1005-1012, DOI: 10.1016/s0003-9993(99)90052-7.

6. Piva SR. Associates of physical function and pain in patients with patellofemoral pain syndrome. Archives of Physical Medicine and Rehabilitation. 2009, 90(2), 285-295. DOI: 10.1016/j. apmr.2008.08.214.

7. Slade PD. What is body image? Behaviour Research and Therapy. 1994, 32(5), 497-502. DOI: 10.1016/0005-7967(94)90136-8.

8. Taleporos G, McCabe MP. Body image and physical disability - personal perspectives. Social Science \& Medicine. 1982, 54(6), 971-980. https://www.sciencedirect.com/science/article/abs/ pii/S0277953601000697.

9. Cash TF. The situational inventory of body-image dysphoria: Psychometric evidence and development of a short form. The International Journal of Eating Disorders. 2002, 32(3), 362366. DOI: 10.1002/eat.10100.

10. Evers L, Verbanck P. Creation of a body satisfaction and global self-perception questionnaire: the QSCPGS. Norms and First Validation. 2010, 36(1), 21-27. DOI: 10.1016/j.encep.2008.12.008.

11. Etchells E, Campus S. Aid to capacity evaluation (ACE). University of Toronto, Joint Centre for Bioethics: Toronto, ON. 2008. http://jcb.utoronto.ca/tools/documents/ace.pdf

12. Brislin RW. Translation and content analysis of oral and written materials. In: Handbook of crosscultural psychology: methodology. H.C. Triandis, J.W. Berry (eds.), 1980, 2; 389-444. https://ci.nii.ac.jp/naid/10030056788/

13. Ware JE, Sherbourne CD. The MOS 36-item short-form health survey (SF-36). I. Conceptual framework and item selection. Medical Care. 1992, 30(6), 473-483. https://www.jstor.org/ stable/3765916? \&seq=1

14. Alonso J, Prieto L, Antó JM. The Spanish version of the SF-36 health survey (the SF-36 health questionnaire): an instrument for measuring clinical results. Medicina Clinica (Barcelona). 1995, 104(20), 771-776. https://europepmc.org/article/med/7783470

15. Vilagut G. The Spanish version of the short form 36 health survey: a decade of experience and new developments. Gaceta Sanitaria. 2005, 19(2), 135-150. DOI: 10.1157/13074369.

16. Muthén LK, Muthén BO. Mplus user's guide. Muthén \& Muthén: Los Angeles, CA. 2011. https:// www.statmodel.com/download/usersguide/Mplus\%20user\%20guide\%20Ver_7_r3_web.pdf

17. Hair JF, Black WC, Babin BJ, Anderson RE, Tatham RL. Multivariate data analysis. Prentice Hall: Upper Saddle River, NJ. 1998; 5. https://pdfs.semanticscholar.org/6885/ bb9a29e8a5804a71bf5b6e813f2f966269bc.pdf

18. Byrne BM. Structural equation modeling: perspectives on the present and the future. International Journal of Testing. 2001, 1(3-4), 327-334. https://www.tandfonline.com/doi/ pdf/10.1080/15305058.2001.9669479? casa_token=nyQB1993hoUAAAAA:lorL1dg4NPIa Ndo-pCHuQ32-voncIe4W3n_V5iD5AsvPGeTpxa1Sv-rwN6s48psGyu8lJR1IeMyX

19. Jöreskog KG, Sörbom D. LISREL 8: User’s reference guide. Scientific Software International. 1996. https://searchworks.stanford.edu/view/4522581

20. Koo TK, Li MY. A guideline of selecting and reporting intraclass correlation coefficients for reliability research. Journal of Chiropractic Medicine. 2016, 15(2), 155-163. DOI: 10.1016/j. jcm.2017.10.001.

21. Schwenk M, Gogulla S, Englert S, Czempik A, Hauer K. Test-retest reliability and minimal detectable change of repeated sit-to-stand analysis using one body fixed sensor in geriatric patients. Physiological Measurement. 2012, 33(11), 1931-1946. DOI: 10.1088/0967-3334/33/11/1931. 
22. Hunt S, McEwen J, McKenna S. The Nottingham health profile: user's manual. Social Science \& Medicine. 1981. DOI: 10.1016/0271-7123(81)90005-5.

23. EuroQol Group. EuroQol - a new facility for the measurement of health-related quality of life. Health Policy. 1990, 16(3), 199-208. DOI: 10.1016/0168-24.8510(92)90164-7

24. Vanti C, et al. Cross-cultural adaptation and validation of the physical therapy outpatient satisfaction survey in an Italian musculoskeletal population. BMC Musculoskeletal Disorders. 2013, 14(1), 125. DOI: 10.1186/1471-2474-14-125.

25. Kahraman T, GençandEGöz A. The Nordic musculoskeletal questionnaire: cross-cultural adaptation into Turkish assessing its psychometric properties. Disability and Rehabilitation. 2016, 38(21), 2153-2160. DOI: 10.3109/09638288.2015.1114034.

26. Dhippayom JP, Trevittaya P, Cheng ASK. Cross-cultural adaptation, validity, and reliability of the patient-rated Michigan hand outcomes questionnaire for Thai patients. Occupational Therapy International. 2018, 6. DOI: 10.1155/2018/8319875.

\section{Annex I - Cross-cultural validation procedure}

First, two bilingual native Spanish translators, specialized in health-related research, translated the questionnaire independently.

Second, the researchers met agreed on a first draft of the QSCPGSe (Spanish version). This draft was later back-translated from Spanish to French by another two independent, native French translators, also specialized in health-related research. Both the translation and back-translation were verified by 5 health professionals, namely, 2 bilingual physiotherapists and 3 bilingual physicians, ensuring conceptual equivalences with the questionnaire's original version.

Third, we pilot tested the QSCPGSe in 30 subjects with non-specific musculoskeletal disorders. After completing the questionnaire, respondents provided feedback regarding possible comprehension difficulties or doubtful aspects the questionnaire. Subsequently, several meetings were held between researchers and bilingual physicians to contrast these observations, both in terms of the possible difficulty in understanding the concepts and the explanatory heading for correct completion.

Most of the difficulties or doubtful aspects were not related to the items' translation, just a few as described here. For example, in the initial heading giving precise instructions, the literal translation of the heading was amended: "Place yourself at the figure, between the two proposals, corresponding to the state which best describes you at this time", by "Please indicate your state of health at this very moment by completing the following scale. Please mark one option for each item.", since 3 people had not understood it and marked levels in both directions.

The concepts "strong" and "very strong" were also amended to "much" and "very much". As for the questionnaire itself, the problem mainly affected three concepts: "bold" that was agreed to be amended to "courageous"; "entirely free" or "entirely without freedom" to "free" and "without freedom"; "source of pleasure" or "source of dislike" to "that pleases" and "that is disliked". Ultimately, the final version of the QSCPGSe was agreed upon the researchers. 


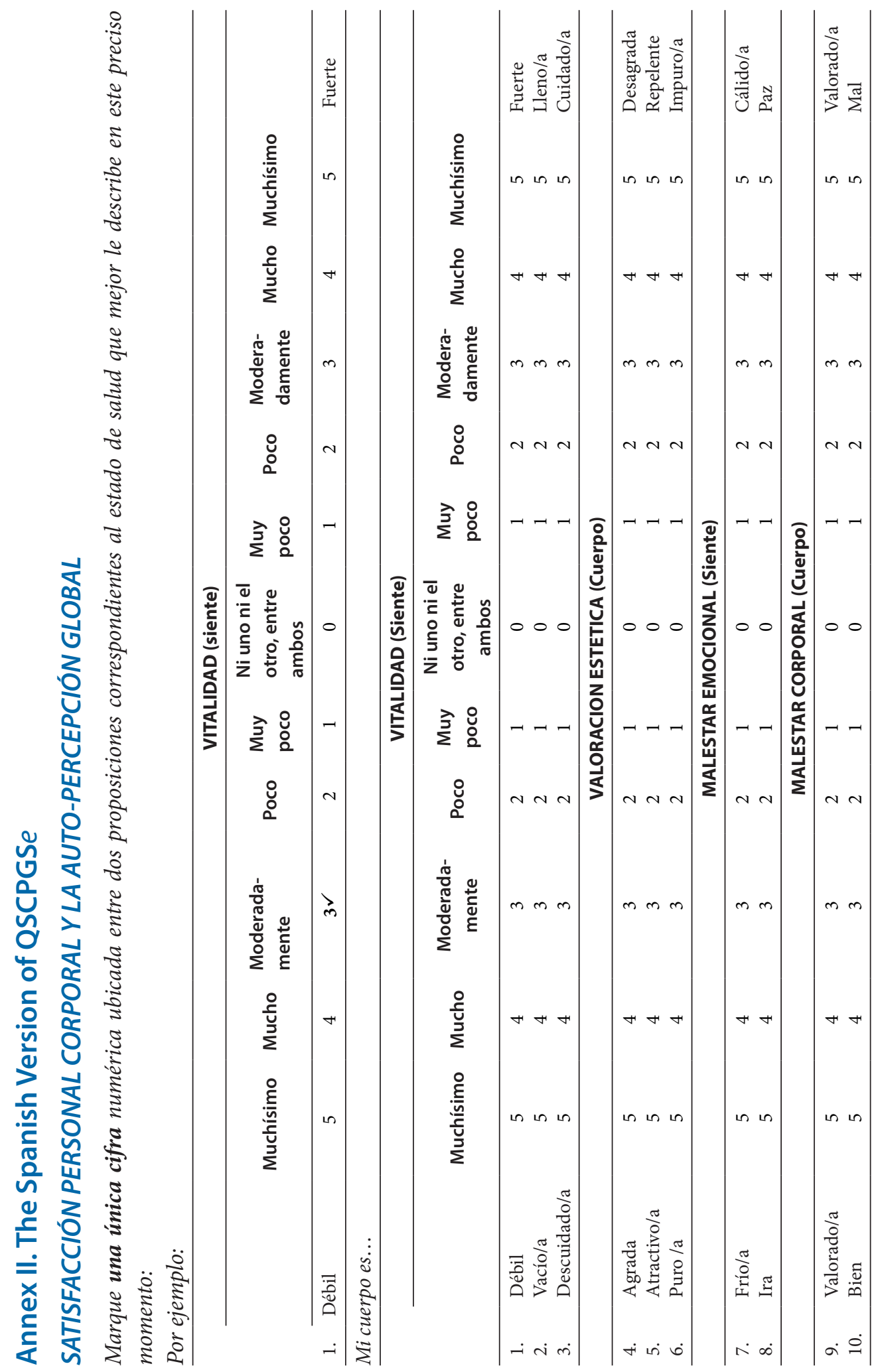




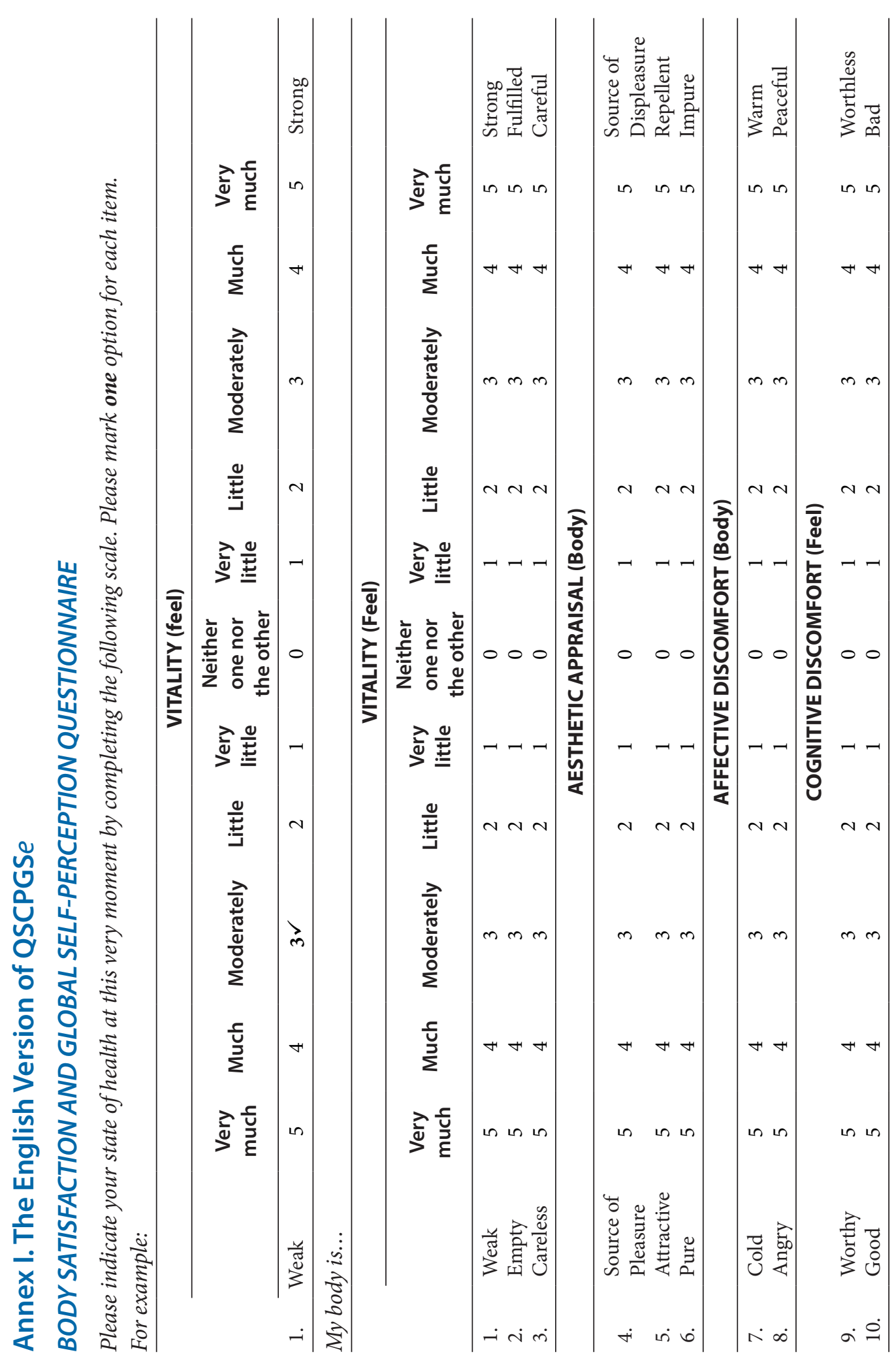

